\title{
miR-196a2 polymorphisms and susceptibility to cancer: A meta-analysis involving 24,697 subjects
}

\author{
PINGYU WANG ${ }^{1}$, SHUYANG XIE $^{2}$, AIDONG CUI $^{3}$, YANXIANG ZHANG $^{2}$ and BAOFA JIANG ${ }^{1}$ \\ ${ }^{1}$ School of Public Health, Shandong University, Jinan; ${ }^{2}$ Binzhou Medical University, Yantai; \\ ${ }^{3}$ Laiyang Central Hospital of Weifang Medical College, Yantai, P.R. China
}

Received September 17, 2011; Accepted November 22, 2011

DOI: 10.3892/etm.2011.399

\begin{abstract}
An increasing number of studies have shown that the hsa-miR-196a2 rs11614913 polymorphism occurs in different types of cancer, but the results are generally controversial and inadequate, mainly due to limited statistical power. To resolve this issue, the present meta-analysis was carried out. Databases, including PubMed and Embase, were searched using: (miR-196a2[All Fields] OR rs11614913[All Fields]) AND ('neoplasms' [MeSH Terms] OR 'neoplasms'[All Fields] OR 'cancer' [All Fields]). Crude odds ratios (ORs) with 95\% confidence intervals (CIs) were summarized in forest plots and detailed in tables. A total of 20 studies, including 11,004 cases and 13,693 controls, were included in the meta-analysis. The hsa-miR-196a2 rs11614913 polymorphism was significantly associated with an increased cancer risk in all genetic models (CC vs. TT: OR=1.280, 95\% CI 1.131-1.449, P<0.001; CT vs. TT: OR=1.187, 95\% CI 1.079-1.306, P<0.001; CC/CT vs. TT: $\mathrm{OR}=1.216,95 \%$ CI 1.104-1.341, $\mathrm{P}<0.001$; and CC vs. CT/TT: $\mathrm{OR}=1.115,95 \%$ CI $1.025-1.213, \mathrm{P}=0.011)$. In conclusion, this meta-analysis provides compelling evidence that the hsa-miR$196 a 2$ rs11614913 polymorphism plays a crucial role in the development of cancer. Screening of patients for the hsa-miR$196 a 2$ rs11614913 polymorphism can prove clinically useful for the prediction and prevention of cancer.
\end{abstract}

Correspondence to: Dr Baofa Jiang, School of Public Health, Shandong University, 44 Wenhua Xi Road, Jinan, Shandong 250012, P.R. China

E-mail: bjiang@sdu.edu.cn

Abbreviations: miRNAs, microRNAs; SNPs, single nucleotide polymorphisms; OR, odds ratio; CI, confidence interval; HWE, Hardy-Weinberg equilibrium; PCR-RFLP, polymerase chain reactionrestriction fragment length polymorphism; PCR-LDR, polymerase chain reaction-ligation detection reaction; HCC, hepatocellular carcinoma; HB, hospital-based healthy controls; PB, population-based controls; ANXA1, Annexin A1

Key words: cancer, hsa-miR-196a2, polymorphism, meta-analysis

\section{Introduction}

During the past several years, extensive effort has been invested in the field of microRNA (miRNA) polymorphisms and the risk of various types of human cancers. miRNAs, originally discovered in Caenorhabditis elegans in 1993 (1) and first reported in 2001 (2), are small, evolutionarily conserved, single-strand non-coding RNA molecules $\sim 22$ nucleotides in length $(3,4)$. It is predicted that miRNAs account for $1-5 \%$ of the human genome and regulate at least $30 \%$ of protein-coding genes and play a crucial role in cancer (5-7). To date, more than one thousand human miRNAs have been identified. Facilitated by continuing technological advances, to date, most registered miRNAs have been widely studied and the results show that both loss and gain of specific miRNA function contribute to cancer development, shedding more light on cancer prevention, diagnosis, progression and outcome.

hsa-miR-196a2, discovered by Lagos-Quintana et al (8), was initially reported to be a prognostic biomarker for non-small cell lung cancer by $\mathrm{Hu}$ et al (9). Since then, emerging molecular epidemiological studies have reported the association between the hsa-miR-196a2 polymorphism and susceptibility to diverse types of human cancer (10-34). Although the precise processes controlling miRNA genetic variants in cancer susceptibility are largely unknown, the importance of miRNA SNPs has been implicated in many cancers. Common single-nucleotide polymorphisms (SNPs), such as hsa-miR-196a2 rs11614913 which is located in the premiRNA, may affect the expression and function of mature miRNAs, resulting in diverse functional consequences, thus opening up a new door through which to explore novel molecular mechanisms of cancer development.

These studies have shown that the hsa-miR-196a2 rs11614913 polymorphism occurs in different types of cancer, but the results are generally controversial and inadequate. In addition, the sample size in each study was relatively small, with statistical power too low to detect the association between the hsa-miR-196a2 polymorphism and cancer risk. To solve the problem of inadequate statistical power and controversial results, it is necessary to carry out a systematic review and meta-analysis to improve our current understanding of the association of the hsa-miR-196a2 rs11614913 polymorphism with human cancer risk. 


\section{Subjects and methods}

Search strategy. The databases, including PubMed and Embase, were searched using: (miR-196a2[All Fields] OR rs11614913[All Fields]) AND ('neoplasms'[MeSH Terms] OR 'neoplasms'[All Fields] OR 'cancer'[All Fields]). The search was restricted to case-control studies published in English and Chinese updated to July 1, 2011. All of the searched studies were reviewed and a manual search of citations from the original studies was performed to identify additional relevant articles.

Inclusion and exclusion criteria. Study quality was assessed according to the proposed checklist of Little et al (35) for reporting and appraising studies of genotype prevalence and gene-disease associations. A study in which all or most of the criteria specified are satisfied would be graded as high quality. The specified inclusion criteria for this meta-analysis were the following: i) case-control studies: cases were patients newly diagnosed and histopathologically confirmed with different types of cancer, while controls were without cancer; ii) evaluation of the association between the rs11614913 and cancer risks; iii) correct statistical analysis and sufficient published data for estimating odds ratio (OR) with $95 \%$ confidence interval (CI); and iv) HardyWeinberg equilibrium (HWE). Animal studies, pure cell studies, studies not concerned with cancer risk, repeated or overlapping studies, studies without complete rs11614913 polymorphism distribution data and studies not fit for HWE were excluded.

Data extraction. Two investigators independently extracted data using standardized forms. When an agreement was not reached, a third investigator resolved the conflict.

The following characteristics were extracted from each study if available: i) first name of the author; ii) publication year; iii) country or region of origin; iv) ethnicity (different ethnic descents were categorized as Caucasian and Asian); v) genotyping method; vi) source of control (population- or hospital-based controls); vii) cancer type; viii) numbers of cases and controls with miR-196a2 rs11614913 CC, CT and TT genotypes, respectively; and ix) P-value for HWE.

Statistical analysis. Based on the complete hsa-miR-196a2 rs11614913 polymorphism distribution data in cases and controls, the crude ORs with their 95\% CIs were performed and displayed as forest plots to assess the strength of association between the hsa-miR-196a2 rs11614913 polymorphism and susceptibility to cancer. The pooled ORs were calculated for homozygote comparison (CC vs. TT), heterozygote comparison (CC vs. CT), dominant model (CC vs. CT/TT) and recessive model (CC/CT vs. TT), respectively. The significance of the pooled OR was determined by the $\mathrm{Z}$-test, and $\mathrm{P}<0.05$ was considered to denote statistical significance. Subgroup analyses were performed for specific cancer types, genotypes, control sources and ethnicities.

Heterogeneity of the study was explored by using both Cochran Q statistic and estimating $\mathrm{I}^{2}$ test (36). When the presence of heterogeneity was detected (P-value $<0.10$ for the Q-test, $\mathrm{I}^{2}$ values $>50 \%$ ), the random effects model (DerSimonian Laird) was chosen. Otherwise, the fixed effects model (Mantel-Haenszel method) was appropriately used to calculate the pooled OR. HWE in the control group was assessed by the Chi-square test for goodness of fit using a

\section{3 records were screened from PubMed}

and 22 from Embase up to 1,July,2011

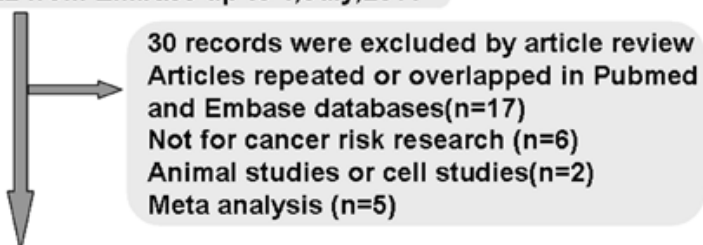

25 full texts and the reference lists were read

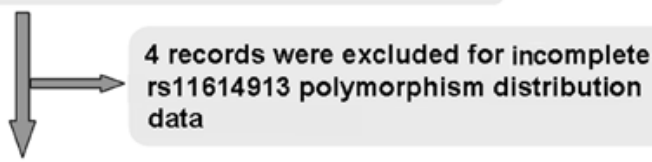

For the remaining $\mathbf{2 1}$ records, study quality

was assessed ,especially Hardy-Weinberg

equilibrium was assessed by chi-square test

2 records were excluded for disagreement with Hardy-Weinberg equilibrium $(P<0.05)$

\section{9 eligible articles finally included}

Figure 1 . The primary studies identified with criteria for inclusion and exclusion in this meta-analysis.

web-based program (http://ihg.gsf.de/cgi-bin/hw/hwa1.pl); $\mathrm{P}<0.05$ was considered significant.

Sensitivity analysis was carried out to assess the stability of the results. A single study involved in the meta-analysis was deleted each time to reflect the influence of the ORs. Publication bias was assessed using Begg and Egger's formal statistical test (statistical significance was defined as $\mathrm{P}<0.10)(37,38)$. Statistical analyses were conducted with Stata 10.0 (Stata Corp., College Station, TX, USA), using two-sided P-values. Meta-analysis was performed using the 'metan' and 'metabias' STATA command.

\section{Results}

Characteristics of the included studies. A total of 55 studies were considered to be relevant by literature search from the PubMed and Embase databases (Fig. 1). Thirty studies were excluded by article review, including 17 studies repeated or overlapped in the PubMed and Embase databases; 6 studies were not concerned with cancer risk research, 1 study was an animal study, 1 study was a cell study, and 5 studies were meta-analysis. During the reading of the 25 full-text manuscripts, 4 studies were excluded due to incomplete rs 1614913 polymorphism distribution data required for OR calculation (10-13). For the remaining 21 records, baseline characteristics of the patients and control subjects were summarized, the study quality was assessed, HWE in particular was assessed by Chi-square test; two records involving Indian populations published in 2011 were excluded for disagreement with HWE $(\mathrm{P}<0.05)(14,15)$, thus leaving 19 articles identified with criteria for inclusion and exclusion (16-34). All studies were case-control studies. The cases were patients with cancer, and the controls were without cancer. The reported age and gender distributions were also recorded. By quality assessment, these studies satisfied most of the criteria specified by Little et al (35). In the study of Catucci et al (20), the genotype 


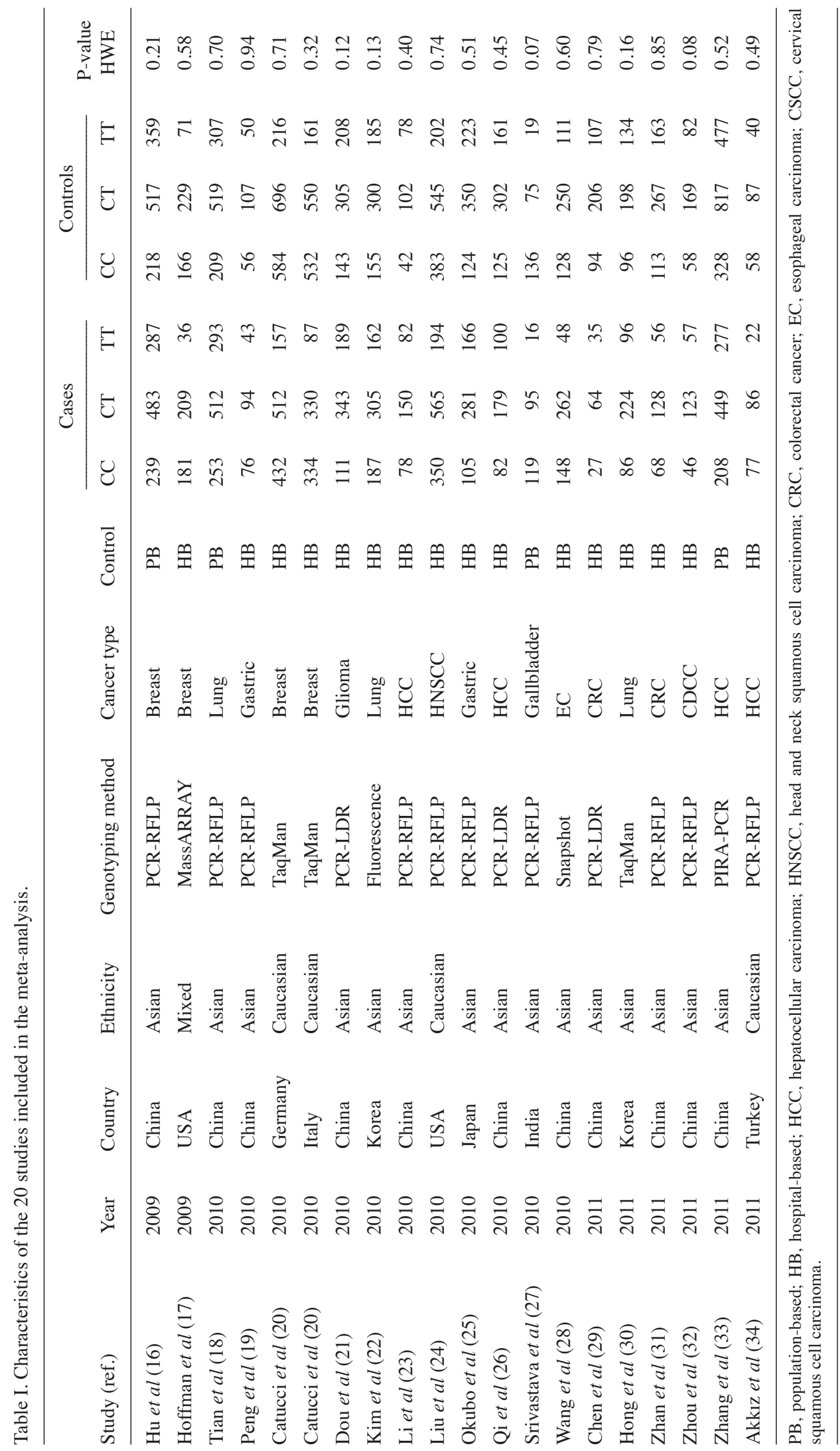




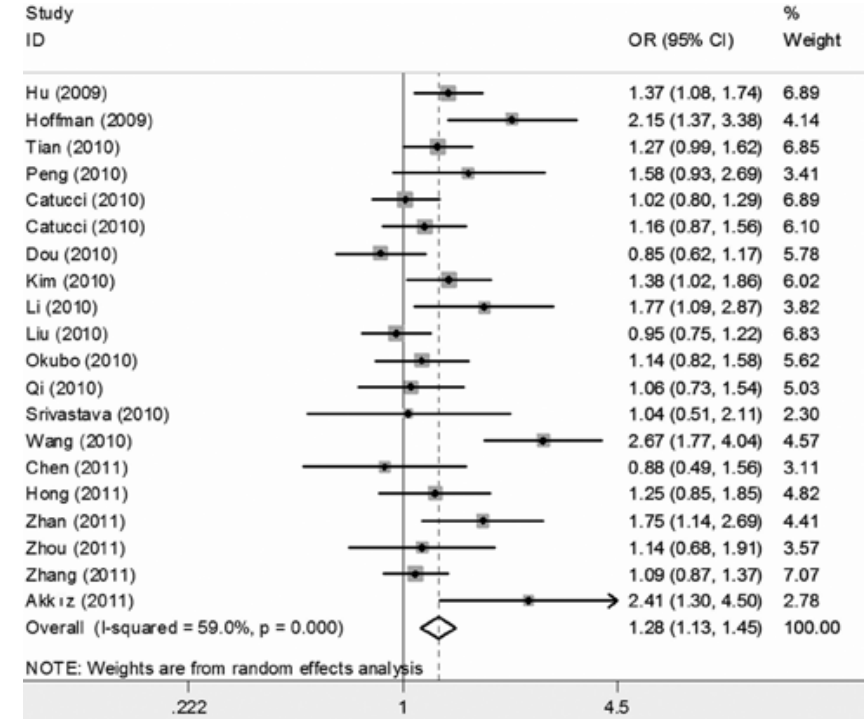

Figure 2. ORs and 95\% CIs for the hsa-miR-196a2 rs11614913 polymorphism (CC vs. TT) in cancers. OR, odds ratio; CI, confidence interval.

frequencies were presented separately for a German and an Italian group, thus each group in the study was considered separately for meta-analysis. Therefore, a total of 20 studies including 11,004 cases and 13,693 controls were included in the meta-analysis.

Table I shows the characteristics of the 20 studies, including first name of the author, year of publication, country of origin, ethnicity, genotyping method, source of controls, cancer type, numbers of cases and controls with miR-196a 2 rs11614913 CC, CT and TT genotypes, respectively and P-value for HWE. Among 20 studies, 2 studies were published in 2009, 12 studies were published in 2010, and 6 studies were published in 2011 . There were 15 studies of Asians, 4 studies of Caucasians, and 1 study of mixed population with no detailed data on ethnicity. Multiple genotyping methods were employed in the studies included in our analysis: 10 studies using polymerase chain reaction-restriction fragment length polymorphism (PCR-RFLP), 3 studies using TaqMan SNP genotyping assay, 3 studies using polymerase chain reaction-ligation detection reaction (PCR-LDR), others using MassARRAY multiplex, and DNA sequencing. A blood sample was used for genotyping in all the studies. There were 4 breast cancer studies, 4 hepatocellular carcinoma (HCC) studies, 3 lung cancer studies, and other cancer types. The controls of 16 studies mainly came from a hospital-based healthy population (HB) matched for gender and age, and 4 studies had population-based controls (PB). The distribution of genotypes in the controls of all of the studies was in agreement with HWE $(\mathrm{P}>0.05)$.

Main meta-analysis results. When all studies were pooled into the meta-analysis, the hsa-miR-196a2 rs11614913 polymorphism was significantly associated with an increased cancer risk in all genetic models (CC vs. TT: OR $=1.280 ; 95 \% \mathrm{CI}$ 1.131-1.449, $\mathrm{P}<0.001$; CT vs. TT: OR $=1.187,95 \%$ CI 1.079 1.306, $\mathrm{P}<0.001 ; \mathrm{CC} / \mathrm{CT}$ vs. TT: $\mathrm{OR}=1.216 ; 95 \%$ CI 1.104-1.341, $\mathrm{P}<0.001$; and CC vs. CT/TT: $\mathrm{OR}=1.115,95 \%$ CI 1.025-1.213, $\mathrm{P}=0.011)$ (Figs. 2 and 3).

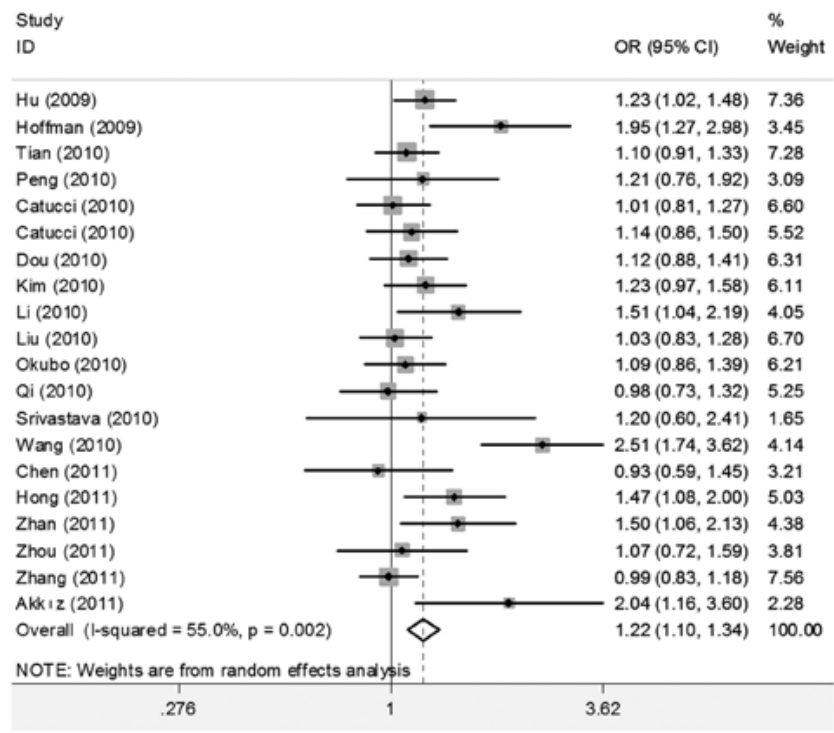

Figure 3. ORs and 95\% CIs for the hsa-miR-196a2 rs11614913 polymorphism (CC/CT vs. TT) in cancers. OR, odds ratio; CI, confidence interval.

Next we performed the subgroup analysis of different specific cancer types, genotypes, control sources and ethnicities (Table II). In the different cancer types, individuals carrying the $\mathrm{CC}$ genotype had an elevated risk of breast cancer (CC vs. TT: $\mathrm{OR}=1.305,95 \%$ CI 1.012-1.684, $\mathrm{P}=0.041$; and $\mathrm{CC}$ vs. CT/TT: OR=1.114, 95\% CI 1.011-1.227, $\mathrm{P}=0.029)$, lung cancer (CC vs. TT: $\mathrm{OR}=1.299,95 \%$ CI 1.096-1.540, $\mathrm{P}=0.003$; and $\mathrm{CC}$ vs. $\mathrm{CT} / \mathrm{TT}$ : $\mathrm{OR}=1.791,95 \% \mathrm{CI} 1.022-1.360, \mathrm{P}=0.024)$, digestive system cancer including gastric and CRC (CC vs. TT: $\mathrm{OR}=1.292,95 \%$ CI 1.041-1.603, $\mathrm{P}=0.020$; and $\mathrm{CC}$ vs. CT/TT: $\mathrm{OR}=1.215,95 \%$ CI $1.015-1.455, \mathrm{P}=0.034)$ and $\mathrm{HCC}(\mathrm{CC}$ vs. CT/ TT: $\mathrm{OR}=1.200,95 \%$ CI 1.038-1.387, $\mathrm{P}=0.014)$ compared with those with the TT or TC/TT genotypes. In addition, individuals carrying the $\mathrm{CT}$ genotype had an elevated risk for breast cancer (CT vs. TT: OR=1.151, 95\% CI 1.012-1.310, $\mathrm{P}=0.032$ ) and other cancers (CT vs. TT: OR $=1.352,95 \%$ CI 1.008 $1.814, \mathrm{P}=0.044$ ) compared with those with the TT genotype. Individuals carrying the $\mathrm{CC} / \mathrm{CT}$ genotype had an elevated risk of lung cancer (CC/CT vs. TT: OR=1.206, 95\% CI 1.054-1.380, $\mathrm{P}=0.007$ ) compared with those with the TT genotype.

In the different genotypes, the hsa-miR-196a2 rs11614913 polymorphism was associated with a significantly increased cancer risk in all genetic models by PCR-RELP (CC vs. TT: $\mathrm{OR}=1.318,95 \%$ CI 1.127-1.541, $\mathrm{P}=0.001$; CT vs. TT: $\mathrm{OR}=1.410,95 \%$ CI $1.038-1.252, \mathrm{P}=0.006 ; \mathrm{CC} / \mathrm{CT}$ vs. TT: $\mathrm{OR}=1.181,95 \%$ CI 1.081-1.290, $\mathrm{P}<0.001$; and $\mathrm{CC}$ vs. CT/TT: $\mathrm{OR}=1.170,95 \%$ CI $1.014-1.350, \mathrm{P}=0.032$ ), and others (CC vs. TT: $\mathrm{OR}=1.662,95 \%$ CI 1.113-2.482, $\mathrm{P}=0.013$; CC/CT vs. TT: $\mathrm{OR}=1.518,95 \%$ CI 1.012-2.278, $\mathrm{P}=0.044$; and CC vs. CT/TT: $\mathrm{OR}=1.237,95 \%$ CI 1.097-1.395, $\mathrm{P}=0.001)$, but no significant associations were observed by PCR-LDR and TaqMan.

In Asian, but not Caucasian ethnicity, significantly increased risks were observed in all genetic models (CC vs. TT: $\mathrm{OR}=1.283$, 95\% CI 1.120-1.470, P<0.001; CT vs. TT: OR=1.187, 95\% CI 1,058-1.331, $\mathrm{P}=0.003$; CC/CT vs. TT: $\mathrm{OR}=1.214,95 \% \mathrm{CI}$ $1.088-1.354, \mathrm{P}=0.001$; and $\mathrm{CC}$ vs. $\mathrm{CT} / \mathrm{TT}: \mathrm{OR}=1.129,95 \% \mathrm{CI}$ 1.019-1.251, $\mathrm{P}=0.021)$. 


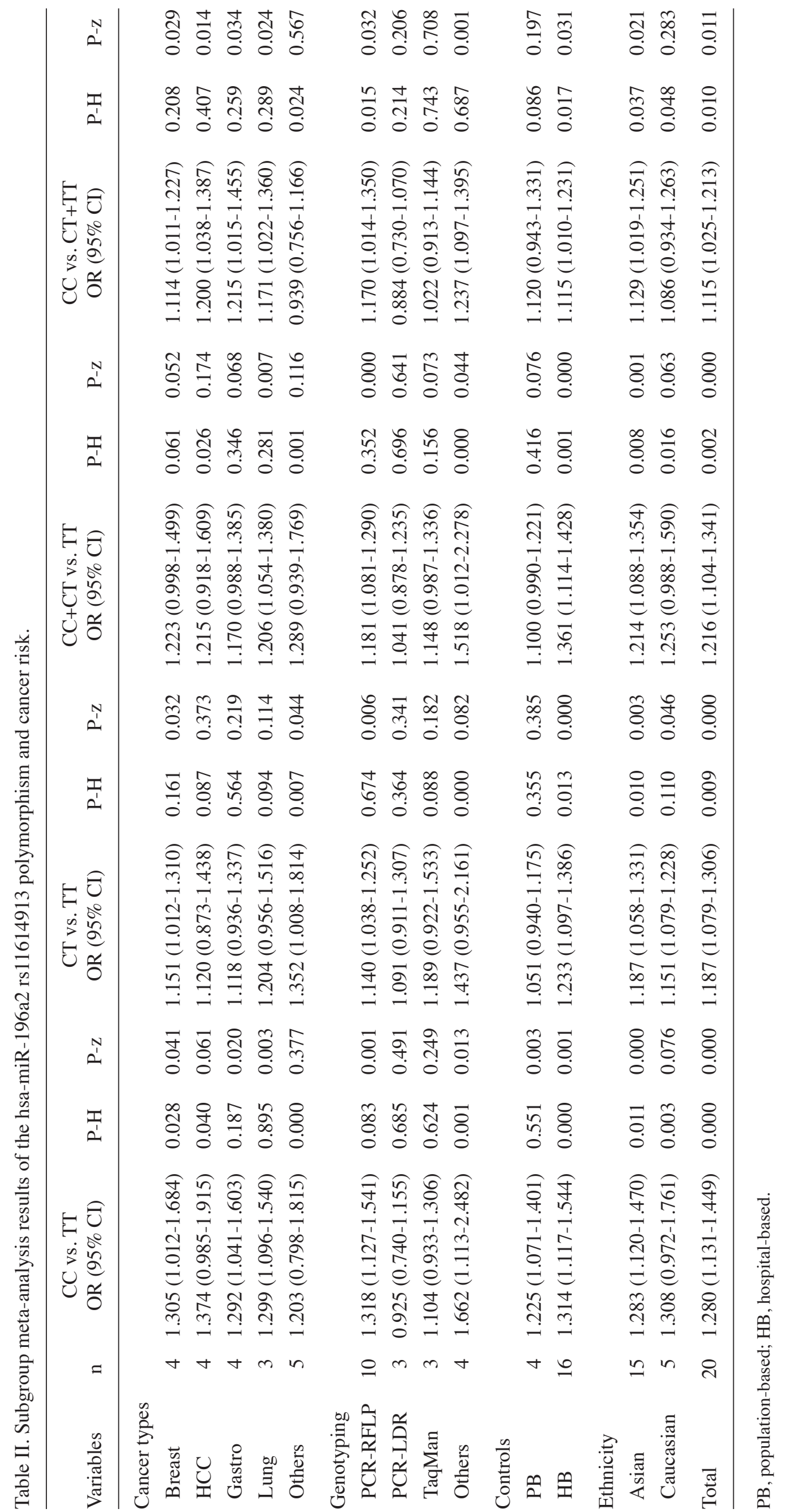


Hospital-based studies demonstrated a significantly increased risk in all genetic models (CC vs. TT: OR $=1.314,95 \%$ CI 1.117 1.544, $\mathrm{P}=0.001$; CT vs. TT: $\mathrm{OR}=1.233 ; 95 \%$ CI $1.097-1.386$, $\mathrm{P}<0.001 ; \mathrm{CC} / \mathrm{CT}$ vs. TT: OR $=1.361,95 \%$ CI $1.114-1.428$, $\mathrm{P}<0.001$; and CC vs. CT/TT: OR=1.115, 95\% CI 1.010-1.231, $\mathrm{P}=0.031$ ). Population-based studies demonstrated significantly increased risks only for the $\mathrm{CC}$ genotype when compared with the TT genotype $(\mathrm{OR}=1.225,95 \% \mathrm{CI} 1.071-1.401, \mathrm{P}=0.003)$.

Sensitivity analysis and publication bias. The overall results in the random model or fixed model were similar. In addition, sensitivity analysis was also carried out by deleting a single study in the meta-analysis each time. The results showed that no individual study affected the overall OR dominantly (data not shown). There was no evidence for publication bias according to Begg's $(\mathrm{z}=1.40, \mathrm{P}=0.163)$ and Egger's tests $(\mathrm{t}=1.32$, $\mathrm{P}=0.202$ ) for $\mathrm{CC}$ vs. $\mathrm{CT} / \mathrm{TT}$.

\section{Discussion}

Principal findings. In the present meta-analysis including 11,004 cases and 13,693 controls, we found that the hsa-miR-196a2 rs11614913 polymorphism was associated with significantly increased overall cancer risk in all genetic models. This metaanalysis provides compelling evidence that the hsa-miR-196a2 rs11614913 polymorphism may play a crucial role in the development of cancer and may be used as a candidate biomarker for cancer susceptibility. Moreover, in our subgroup analysis, we indicated that individuals carrying the $\mathrm{CC}$ genotype had a significantly elevated risk of breast cancer, lung cancer, digestive system cancer (including gastric and CRC) and HCC compared with those with TT or TC/TT genotypes, consistent with the total results. Ryan et al (39) suggested that variations in miRNAs may be related to the risk of cancer and reported that the rs11614913 polymorphism located in the hsa-miR-196a2 3' mature sequence affects the maturation and may affect target mRNA. Cell culture experiments also indicated that high hsa-miR-196a levels could suppress the activities of various cancer-related genes, such as ANXA1 (Annexin A1), suppression of which is well documented in various cancer types $(40,41)$. Li et al $(23)$ conducted an analysis of rs11614913 genotypes and the expression of mature miR-196a. They found that the expression level of hsa-miR-196a was significantly higher in CC patients or patients carrying at least one $\mathrm{C}$ allele than in TT patients. The $\mathrm{CC}$ homozygotes were associated with a statistically significant increase in mature miR196a. Therefore, the altered expression patterns of miR-196a influence its potential targets and may play a role in the regulatory processes that occur during cancer development.

Over the past several years, a large number of distinct genotyping approaches have been designed for SNP detection and identification, typically involving the amplification of the target DNA sequence and the detection of SNPs, but each genotyping method has its merit and demerit. A proper technology platform should be adopted according to the sample size and the amount of SNPs (42). The results of PCR-RFLP, PCR-LDR, TaqMan and other methods to detect the hsa-miR-196a2 rs11614913 polymorphism may not be in full accord. We found that the hsamiR-196a2 rs11614913 polymorphism significantly increased cancer risk in all genetic models using the PCR-RFLP method, but not using the PCR-LDR method. Therefore, a great deal of effort should be devoted to developing more accurate, rapid, and cost-effective technologies for SNP analysis.

In the subgroup analysis of source of controls and ethnicities, hospital-based and population-based studies demonstrated significantly increased risks for the $\mathrm{CC}$ genotype compared with the TT genotype. A population-based control can better represent the population, but hospital-based controls are more readily obtainable in research. In addition, significantly increased risks were observed in an Asian but not in a Caucasian ethnic population, suggesting potentially different mechanisms in different populations according to different genetic background and environment. More studies in other ethnic groups may be necessary for further progress in this area.

Strengths and limitations of the meta-analysis. Several limitations of this meta-analysis should be mentioned. First, the meta-analysis was limited by a relatively small number of available studies. It is difficult to perform subgroup analysis for every type of cancer. Second, our analysis was limited to Asian and Caucasian ethnicities, so it is uncertain whether these results are generalizable to other populations. Third, restriction to studies published in English or Chinese may confer potential language bias. In addition, studies with no statistically significant results often have less chance for publication. It is still difficult to rule out potential publication bias in the meta-analysis. To confirm the role of the hsa-miR-196a2 rs11614913 polymorphism in cancer risk requires further larger studies in different populations and in different types of cancer.

In spite of these limitations, our meta-analysis had several strengths. In genetic association studies, the sample size and statistical power are often of particular importance. We overcame the limitations of a single study involving a relative small number of subjects and the limitation in the statistical significance, as relatively more sufficient number of cases and controls were pooled from different studies, which significantly increased the statistical power of the analysis. In addition, this meta-analysis not only assessed the total strength of association between the hsa-miR-196a2 rs11614913 polymorphism and overall cancer risk in all genetic models, but also further performed subgroup analysis of different specific cancer types, genotypes and control sources to assess the polymorphism with cancer risk.

In conclusion, this meta-analysis provides compelling evidence that the hsa-miR-196a2 rs11614913 polymorphism may play a crucial role in the development of cancer, and that screening of patients for the hsa-miR-196a2 rs11614913 polymorphism is clinically useful for the prediction and prevention of cancer.

\section{Acknowledgements}

This study was supported by grants from the NCET-10-0919, National Natural Science Foundation (no. 30801324), the Foundation of Shandong Natural Science (nos. ZR2009CL005 and ZR2009CQ033), and the Scientific Research Foundation of Shandong Educational Committee (nos. J09LF11 and J08LG15).

\section{References}

1. Lee RC, Feinbaum RL and Ambros V: The $C$. elegans heterochronic gene lin encodes small RNAs with antisense complementarity to lin. Cell 75: 843-854, 1993. 
2. Lau NC, Lim LP, Weinstein EG and Bartel DP: An abundant class of tiny RNAs with probable regulatory roles in Caenorhabditis elegans. Science 294: 858-862, 2001.

3. Macfarlane LA and Murphy PR: MicroRNA: biogenesis, function and role in cancer. Curr Genomics 11: 537-561, 2010.

4. Bartel DP: MicroRNAs: genomics, biogenesis, mechanism, and function. Cell 116: 281-297, 2004.

5. Liu W, Mao SY and Zhu WY: Impact of tiny miRNAs on cancers. World J Gastroenterol 13: 497-502, 2007.

6. Davis CD and Ross SA: Evidence for dietary regulation of microRNA expression in cancer cells. Nutr Rev 66: 477-482, 2008.

7. Gao LB, Bai P, Pan XM, Jia J, Li LJ, Liang WB, Tang M, Zhang LS, Wei YG and Zhang L: The association between two polymorphisms in pre-miRNAs and breast cancer risk: a metaanalysis. Breast Cancer Res Treat 125: 571-574, 2011.

8. Lagos-Quintana M, Rauhut R, Meyer J, Borkhardt A and Tuschl T: New microRNAs from mouse and human. RNA 9: $175-179,2003$

9. Hu Z, Chen J, Tian T, Zhou X, Gu H, Xu L, Zeng Y, Miao R, Jin $\mathrm{G}, \mathrm{Ma} H$, Chen $\mathrm{Y}$ and Shen $\mathrm{H}$ : Genetic variants of miRNA sequences and non-small cell lung cancer survival. J Clin Invest 118: 2600-2608, 2008

10. Yang H, Dinney CP, Ye Y, Zhu Y, Grossman HB and Wu X Evaluation of genetic variants in microRNA-related genes and risk of bladder cancer. Cancer Res 68: 2530-2537, 2008.

11. Christensen BC, Avissar-Whiting M, Ouellet LG, Butler RA, Nelson HH, McClean MD, Marsit CJ and Kelsey KT: Mature microRNA sequence polymorphism in MIR196A2 is associated with risk and prognosis of head and neck cancer. Clin Cancer Res 16: 3713-3720, 2010

12. Ye Y, Wang KK, Gu J, Yang H, Lin J, Ajani JA and Wu X: Genetic variations in microRNA-related genes are novel susceptibility loci for esophageal cancer risk. Cancer Prev Res (Phila) 1: 460-469, 2008.

13. Horikawa Y, Wood CG, Yang H, Zhao H, Ye Y, Gu J, Lin J, Habuchi T and Wu X: Single nucleotide polymorphisms of microRNA machinery genes modify the risk of renal cell carcinoma. Clin Cancer Res 14: 7956-7962, 2008.

14. George GP, Gangwar R, Mandal RK, Sankhwar SN and Mittal RD Genetic variation in microRNA genes and prostate cancer risk in North Indian population. Mol Biol Rep 38: 1609-1615, 2011.

15. Mittal RD, Gangwar R, George GP, Mittal T and Kapoor R: Investigative role of pre-microRNAs in bladder cancer patients: a case-control study in North India. DNA Cell Biol 30: 401-406, 2011

16. Hu Z, Liang J, Wang Z, Tian T, Zhou X, Chen J, Miao R, Wang Y, Wang $\mathrm{X}$ and Shen $\mathrm{H}$ : Common genetic variants in pre-microRNAs were associated with increased risk of breast cancer in Chinese women. Hum Mutat 30: 79-84, 2009.

17. Hoffman AE, Zheng T, Yi C, Leaderer D, Weidhaas J, Slack F, Zhang Y, Paranjape T and Zhu Y: MicroRNA miR-196a-2 and breast cancer: a genetic and epigenetic association study and functional analysis. Cancer Res 69: 5970-5977, 2009.

18. Tian T, Shu Y, Chen J, Hu Z, Xu L, Jin G, Liang J, Liu P, Zhou X, Miao R, Ma H, Chen Y and Shen $\mathrm{H}$ : A functional genetic variant in microRNA-196a2 is associated with increased susceptibility of lung cancer in Chinese. Cancer Epidemiol Biomarkers Prev 18: 1183-1187, 2009

19. Peng S, Kuang Z, Sheng $\mathrm{C}$, Zhang $\mathrm{Y}, \mathrm{Xu} \mathrm{H}$ and Cheng Q: Association of microRNA-196a-2 gene polymorphism with gastric cancer risk in a Chinese population. Dig Dis Sci 55: 2288-2293, 2010.

20. Catucci I, Yang R, Verderio P, Pizzamiglio S, Heesen L, Hemminki K, Sutter C, Wappenschmidt B, Dick M, Arnold N, Bugert P, Niederacher D, Meindl A, Schmutzler RK, Bartram CC, Ficarazzi F, Tizzoni L, Zaffaroni D, Manoukian S, Barile M, Pierotti MA, Radice P, Burwinkel B and Peterlongo P: Evaluation of SNPs in miR-146a, miR196a2 and miR-499 as low-penetrance alleles in German and Italian familial breast cancer cases. Hum Mutat 31: E1052-E1057, 2010

21. Dou T, Wu Q, Chen X, Ribas J, Ni X, Tang C, Huang F, Zhou L and $\mathrm{Lu} \mathrm{D}$ : A polymorphism of microRNA196a genome region was associated with decreased risk of glioma in Chinese population. J Cancer Res Clin Oncol 136: 1853-1859, 2010

22. Kim MJ, Yoo SS, Choi YY and Park JY: A functional polymorphism in the pre-microRNA-196a2 and the risk of lung cancer in a Korean population. Lung Cancer 69: 127-129, 2010.
23. Li XD,LiZG, Song XX and Liu CF: A variant in microRNA-196a2 is associated with susceptibility to hepatocellular carcinoma in Chinese patients with cirrhosis. Pathology 42: 669-673, 2010.

24. Liu Z, Li G, Wei S, Niu J, El-Naggar AK, Sturgis EM and Wei Q: Genetic variants in selected pre-microRNA genes and the risk of squamous cell carcinoma of the head and neck. Cancer 116 4753-4760, 2010

25. Okubo M, Tahara T, Shibata T, Yamashita H, Nakamura M, Yoshioka D, Yonemura J, Ishizuka T, Arisawa T and Hirata I: Association between common genetic variants in pre-microRNAs and gastric cancer risk in Japanese Population. Helicobacter 15: 524-531, 2010.

26. Qi P, Dou TH, Geng L, Zhou FG, Gu X, Wang H and Gao CF: Association of a variant in MIR 196A2 with susceptibility to hepatocellular carcinoma in male Chinese patients with chronic hepatitis B virus infection. Hum Immunol 71: 621-626, 2010.

27. Srivastava K, Srivastava A and Mittal B: Common genetic variants in premicroRNAs and risk of gallbladder cancer in North Indian population. J Hum Genet 55: 495-499, 2010.

28. Wang K, Guo H, Hu H, Xiong G, Guan X, Li J, Xu X, Yang K and Bai Y: A functional variation in pre-microRNA-196a is associated with susceptibility of esophageal squamous cell carcinoma risk in Chinese Han. Biomarkers 15: 614-618, 2010.

29. Chen H, Sun LY, Chen LL, Zheng HQ and Zhang QF: A variant in microRNA-196a2 is not associated with susceptibility to and progression of colorectal cancer in Chinese. Intern Med J: Jan 17, 2011 (Epub ahead of print). doi: 10.1111/j.1445-5994 2011.02434.x

30. Hong YS, Kang HJ, Kwak JY, Park BL, You CH, Kim YM and Kim H: Association between microRNA196a2 rs11614913 genotypes and the risk of non-small cell lung cancer in Korean population. J Prev Med Public Health 44: 125-230, 2011.

31. Zhan JF, Chen LH, Chen ZX, Yuan YW, Xie GZ, Sun AM and Liu Y: A functional variant in microRNA-196a2 is associated with susceptibility of colorectal cancer in a Chinese population. Arch Med Res 42: 144-148, 2011

32. Zhou B, Wang K, Wang Y, Xi M, Zhang Z, Song Y and Zhang L: Common genetic polymorphisms in pre-microRNAs and risk of cervical squamous cell carcinoma. Mol Carcinog 50: 499-505, 2011.

33. Zhang XW, Pan SD, Feng YL, Liu JB, Dong J, Zhang YX, Chen JG, Hu ZB and Shen HB: Relationship between genetic polymorphism in microRNAs precursor and genetic prediposition of hepatocellular carcinoma. Zhonghua Yu Fang Yi Xue Za Zhi 45: 239-243, 2011 (In Chinese).

34. Akkız H, Bayram S, Bekar A, Akgöllü E and Ulger Y: A functional polymorphism in pre-microRNA-196a-2 contributes to the susceptibility of hepatocellular carcinoma in a Turkish population: a case-control study. J Viral Hepat 18: e399-e407, 2011.

35. Little J, Bradley L, Bray MS, Clyne M, Dorman J, Ellsworth DL, Hanson J, Khoury M, Lau J, O'Brien TR, Rothman N, Stroup D, Taioli E, Thomas D, Vainio H, Wacholder S and Weinberg C: Reporting, appraising, and integrating data on genotype prevalence and gene-disease associations. Am J Epidemiol 156: 300-310, 2002.

36. Higgins JP, Thompson SG, Deeks JJ and Altman DG: Measuring inconsistency in meta-analyses. BMJ 327: 557-560, 2003.

37. Begg CB and Mazumdar M: Operating characteristics of a rank correlation test for publication bias. Biometrics 50: 1088-1101, 1994

38. Egger M, Davey SG, Schneider M and Minder C: Bias in metaanalysis detected by a simple, graphical test. BMJ 315: 629-634, 1997.

39. Ryan BM, Robles AI and Harris CC: Genetic variation in microRNA networks: the implications for cancer research. Nat Rev Cancer 10: 389-402, 2010.

40. Luthra R, Singh RR, Luthra MG, Li YX, Hannah C, Romans AM, Barkoh BA, Chen SS, Ensor J, Maru DM, Broaddus RR, Rashid A and Albarracin CT: MicroRNA-196a targets annexin A1: a microRNAmediated mechanism of annexin A1 downregulation in cancers. Oncogene 27: 6667-6678, 2008.

41. Shen D, Nooraie F, Elshimali Y, Lonsberry V, He J, Bose S Chia D, Seligson D, Chang HR and Goodglick L: Decreased expression of annexin A1 is correlated with breast cancer development and progression as determined by a tissue microarray analysis. Hum Pathol 37: 1583-1591, 2006.

42. Kim S and Misra A: SNP genotyping: technologies and biomedical applications. Annu Rev Biomed Eng 9: 289-320, 2007. 